\section{Continuous expression of the homeobox gene Pax6 in the ageing human retina}

\begin{abstract}
Purpose In the past few years, the essential role of the homeobox gene Pax6 for eye development has been demonstrated unambiguously in a variety of species including humans. In humans, Pax6 mutations lead to a variety of ocular malformations of the anterior and posterior segment. However, little is known about PAX6 expression in the adult human retina. We have therefore investigated PAX6 levels and localization in the human retina at various ages.

Methods Adult human eyes of various ages (17-79 years) were obtained from the Zurich Eye Bank. PAX6 expression levels and patterns were analysed by Western blot analysis of total retinal protein and by immunohistochemistry on paraffin sections, respectively.

Results PAX6 expression in the retina was detected up to 79 years of donor age and was predominantly localized to the ganglion cell layer and the inner part of the inner nuclear layer.

Conclusions PAX6 remains distinctly expressed throughout the lifespan of the human retina suggesting a role for PAX6 in the retina after completion of eye morphogenesis. Eye (2007) 21, 90-93. doi:10.1038/sj.eye.6702166; published online 28 October 2005
\end{abstract}

Keywords: foveal hypoplasia; PAX6; human; aniridia; Peter's anomaly

\section{Introduction}

The homeobox gene Pax 6 is an essential regulatory element for ocular development in a variety of species with special emphasis on mammals. ${ }^{1}$ The predominant role of Pax6 even in different animal phyla such as insects and
D Stanescu' ${ }^{1}$, HP Iseli', K Schwerdtfeger ${ }^{2}$, LM Ittner ${ }^{2}$, CE Remé ${ }^{1}$ and $\mathrm{F} \mathrm{Hafezi}{ }^{1}$ vertebrates is demonstrated by the high rate of conservation of Pax 6 domains throughout evolution, as demonstrated by a $93 \%$ homology of the PAX6 amino acid sequence between Drosophila and human over the paired domain and the homeodomain. ${ }^{2}$

In humans, Pax 6 has been mapped to chromosome 11p13 and is expressed mainly in the eye, brain, and pancreas during development. In the last years, a number of human Pax6 mutations have been identified leading to a variety of ocular malformations of the anterior and posterior segment. ${ }^{3-11}$

In the developing human retina, PAX6 expression can be histochemically detected in the Inner Nuclear Layer and Ganglion Cell Layer, as demonstrated by Nishina et $a l^{12}$ in human foetuses up to gestation week 22 .

Interestingly, research on expression patterns and regulatory function of PAX6 had focused on developmental stages solely and little is known about the role of PAX6 in the ageing mammalian retina. We have recently shown that in the mouse retina, PAX6 is constantly expressed throughout the whole lifespan of the animal. Furthermore, we have demonstrated that PAX6 is downregulated in two animal models for retinal degeneration during the peak of photoreceptor apoptosis (Iseli et al, submitted). To analyse whether PAX6 expression in the human retina persists after the end of ocular development, we performed Western Blot analyses and immunohistochemical studies on human retinas at various ages.

\section{Materials and methods \\ Dissection of retinas}

Human retinas were obtained from seven eyes from donors aged 17-79 years. Eyes were 
provided by the Zurich Eye Bank as globes with excised cornea, the latter being further processed for corneal grafting. The procurement and use of human tissues in this study complied with the Declaration of Helsinki. Causes of death included myocardial infarction, cerebral vascular accident, renal insufficiency, multiple organ failure, aortic dissection, and congestive heart failure. Time between death and enucleation was below $12 \mathrm{~h}$ and time between death and dissection was below $14 \mathrm{~h}$ in all samples (Table 1). Prior to dissection, the posterior pole was inspected through an operating microscope to confirm the absence of gross retinal disease. The central retina was defined as the region delimited by the optic nerve nasally, the retinal arcuate vessels superiorly and inferiorly and an imaginary line $2 \mathrm{~mm}$ temporally of the fovea. All retinas were dissected on ice. Briefly, corneas were excised and vitreous was extruded with a forceps. Retinas were gently removed from the retinal pigment epithelium (RPE) with a smooth forceps at the ora serrata and the optic nerve head. Care was taken that retinal tissue was free from RPE remnants. Central retinal tissue was frozen in liquid nitrogen and stored at $-80^{\circ} \mathrm{C}$. All samples used in this study were free of malignancies or any known ocular disease.

\section{Western blotting}

Human retinas were homogenized in $100 \mathrm{mM}$ Tris-Hcl, pH 7.4 using a sonifier. Protein concentrations were determined by the Bradford assay using BSA as standard. Western blotting was performed according to standard protocols. For immunodetection, polyclonal rabbit antisera directed against PAX6 were applied..$^{13}$ After application of HRP-conjugated secondary antibodies, immunoreactivity was visualized using the Renaissance-Western blot detection kit (Perkin-Elmer Life Sciences, Emeryville, USA).

\section{Immunohistochemical analysis}

For immunohistochemistry, whole eyecups were fixed by immersion in $4 \%$ paraformaldehyde in $100 \mathrm{mM}$ PBS

Table 1 Human donor information

\begin{tabular}{lllrll}
\hline \multicolumn{2}{l}{ Sample Age (years) } & Sex & TDE $(h)$ & TDF Cause of death \\
\hline 1 & 17 & F & 9 & 13 & Polytrauma \\
2 & 28 & M & 12 & 12 & Polytrauma \\
3 & 36 & M & 5 & 8 & Myocardial infarction \\
4 & 45 & M & 7 & 9 & Myocardial infarction \\
5 & 55 & M & 9 & 13 & Renal failure \\
6 & 66 & M & 6 & 9 & Cerebral vascular accident \\
7 & 79 & F & 8 & 11 & Cerebral vascular accident
\end{tabular}

$\mathrm{GW}=$ gestation week; TDE = time between death and enucleation; $\mathrm{TDF}=$ time between death and fixation.
( $\mathrm{pH}$ 7.4) overnight and processed to paraffin blocks using standard protocols. Sections $(10 \mu \mathrm{m})$ were deparaffinized in xylene and dehydrated in descending ethanol series. To enhance immunoreactivity, sections were incubated in $10 \mathrm{mM}$ citrate buffer $(\mathrm{pH}$ 6.0) and boiled in a steamer at $100^{\circ} \mathrm{C}$ for $3 \mathrm{~min}$. For immunofluorescence primary polyclonal rabbit antibodies for the detection of Pax6 were used at dilutions of 1:300 (Covance, Princeton, NJ, USA) or $1: 200$ (Chemicon, Temecula, CA, USA) overnight at $4{ }^{\circ} \mathrm{C}$, and visualized via biotinylated secondary antibodies (goat anti-rabbit; 1:200; Vector Labs, Burlingame, CA, USA) and streptavidin-Cy3 ( 1 : 200; Sigma-Aldrich, St Louis, MO, USA). Absence of the primary antibody was used as negative control (data not shown).

\section{Results}

Specificity of the antibody was examined by Western blot analysis. Blots display a band with a molecular weight of $46 \mathrm{kDa}$ and a second band of $48 \mathrm{kDa}$ corresponding to the splicing isoform of PAX6, PAX6(5a). As an internal control labelling with an antibody against actin was performed. Actin levels remained constant throughout all time points tested in all experiments performed (data not shown).

Persistent expression was detected at all ages tested: $17,28,36,45,55,66$, and 79 years with varying levels of expression as shown in Figure 1.

Immunohistochemical analysis is shown in Figure 2a and reveals that PAX6 expression in the retina is confined to the ganglion cell layer (GCL) and the inner portion of the inner nuclear layer (INL).

\section{Discussion}

In humans, a number of heterozygous Pax6 mutations has been identified in the last years leading to a large variety of different ocular disorders and malformations of both the anterior and posterior segment. These disorders range from autosomal dominant keratitis, congenital cataract, Peter's anomaly and aniridia to isolated foveal hypoplasia, ${ }^{3-11}$ for review see. ${ }^{14}$ Furthermore, homozygous human Pax6 mutations lead to anophtalmia, brain malformation, and early postnatal

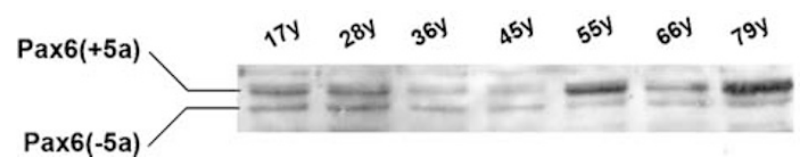

Figure 1 Western blot analysis of PAX6 expression and its alternative splice forms in human retinas at various ages $(17,28$, $36,45,55,66$, and 79 years). Expression was detected at all ages tested with varying levels. 


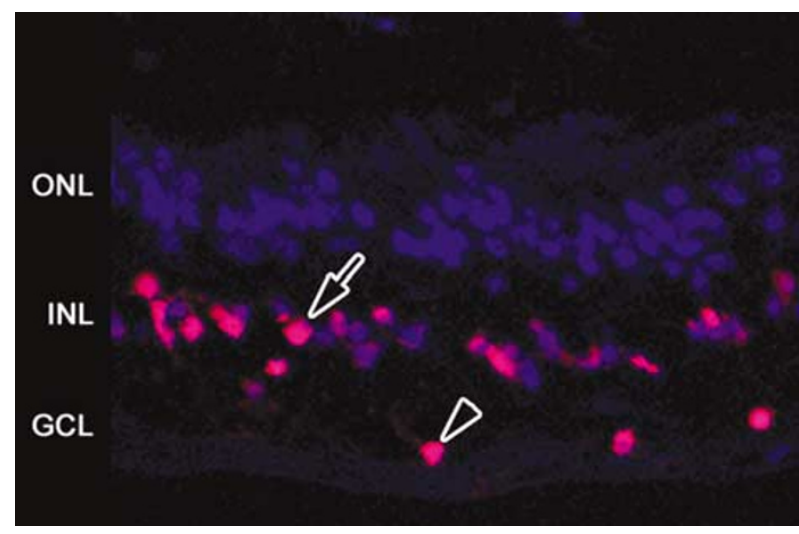

Figure 2 Positive PAX6 immunostaining (a) of nuclei in the INL (arrow) and GCL (arrowhead) of the inner human retina. outer nuclear layer (ONL); inner nuclear layer (INL); ganglion cell layer (GCL).

death. ${ }^{15}$ Little is known about Pax6 expression in the adult human retina: Nishina et $a l^{12}$ have studied PAX6 expression in human foetuses until gestation week 22 and histochemically detected Pax6 expression in the INL and GCL.

Similarly, in mice, homozygous mutations of the Pax 6 homologue small eye (sey) lead to a distinct phenotype with early postnatal death and absence of eyes whereas heterozygous $(s e y /+)$ mutations show microphthalmia and corneal, chamber angle and lenticular abnormalities ${ }^{16,17}$ with striking similarities to the human condition. . $^{5,8,15,18}$

There is growing evidence that the two major isoforms of PAX6, PAX6(+5a) and PAX6(-5a) may play different biological roles: Dominguez et al ${ }^{19}$ have shown lately that in fDrosophila PAX6 $(+5 a)$ functions primarily in the regulation of growth and less effectively in eye specification than Pax6(-5a). Furthermore, the Pax6 $(+5 a)$ isoform is involved in the chain of events leading to formation of the fovea centralis in the developing chick eye whereas Pax6(-5a) is not. ${ }^{20}$ Our human data show expression of both isoforms in the retina with varying levels.

To test whether PAX6 is also expressed in the ageing human retina we have analysed the retinas of six individuals of different ages up to 79 years by Western blot analysis and immunohistochemistry and found a persistent retinal expression of PAX6 with localization to the inner retina, similar to the embryonic ${ }^{12}$ condition. Equal amounts of total retinal protein were loaded as verified by tubulin control blots (data not shown). Nevertheless, PAX6 expression varied markedly between different sample ages. This might either be due to different degrees of protein degradation in post mortem eyes or it might represent interindividual changes in protein expression levels.
The question remains why a master gene of development is expressed in an ageing tissue. To address this question, Walter Gehring and collaborators have performed an experiment in the ribbonworm Lineus where they suppressed PAX6 expression in the adult eye by RNA interference: 37 days after suppression of PAX6, the eyes disappeared (W Gehring, personal communication).

Furthermore, we have recently shown that in wildtype mice, PAX6 expression in the inner retina persists during the whole lifespan of animals. Interestingly, PAX6 is downregulated in two different animal models for retinal degeneration during the peak of retinal apoptosis (Iseli et al, submitted).

In light of the striking similarities of Pax6 mutations in mice and men, our findings in the human retina suggest a potential role for Pax6 in the human retina after completion of eye morphogenesis.

\section{Acknowledgements}

We thank Coni Imsand and Gaby Hoegger for skilled technical assistance, Christian Grimm and Andreas Wenzel for fruitful discussions and continuous support and Walter Gehring for careful reading of the manuscript. This work was supported by Roche Foundation, Basel, Switzerland.

\section{References}

1 Gehring WJ, Ikeo K. Pax 6: mastering eye morphogenesis and eye evolution. Trends Genet 1999; 15(9): 371-377.

2 Quiring R, Walldorf U, Kloter U, Gehring WJ. Homology of the eyeless gene of Drosophila to the Small eye gene in mice and Aniridia in humans. Science 1994; 265(5173): 785-789.

3 Azuma N, Nishina S, Yanagisawa H, Okuyama T, Yamada M. PAX6 missense mutation in isolated foveal hypoplasia. Nat Genet 1996; 13(2): 141-142.

4 Dharmaraj N, Reddy A, Kiran V, Mandal A, Panicker S, Chakrabarti S. PAX6 gene mutations and genotypephenotype correlations in sporadic cases of aniridia from India. Ophthalmic Genet 2003; 24(3): 161-165.

5 Hanson IM, Fletcher JM, Jordan T, Brown A, Taylor D, Adams RJ et al. Mutations at the PAX6 locus are found in heterogeneous anterior segment malformations including Peters' anomaly. Nat Genet 1994; 6(2): 168-173.

6 Jordan T, Hanson I, Zaletayev D, Hodgson S, Prosser J, Seawright A et al. The human PAX6 gene is mutated in two patients with aniridia. Nat Genet 1992; 1(5): 328-332.

7 Lauderdale JD, Wilensky JS, Oliver ER, Walton DS, Glaser T. 3' deletions cause aniridia by preventing PAX6 gene expression. Proc Natl Acad Sci USA 2000; 97(25): 13755-13759.

8 Mirzayans F, Pearce WG, MacDonald IM, Walter MA. Mutation of the PAX6 gene in patients with autosomal dominant keratitis. Am J Hum Genet 1995; 57(3): 539-548.

9 Nanjo Y, Kawasaki S, Mori K, Sotozono C, Inatomi T, Kinoshita S. A novel mutation in the alternative splice 
region of the PAX6 gene in a patient with Peters' anomaly. Br J Ophthalmol 2004; 88(5): 720-721.

10 Vincent MC, Gallai R, Olivier D, Speeg-Schatz C, Flament J, Calvas $\mathrm{P}$ et al. Variable phenotype related to a novel PAX 6 mutation (IVS4 $+5 \mathrm{G}>\mathrm{C}$ ) in a family presenting congenital nystagmus and foveal hypoplasia. Am J Ophthalmol 2004; 138(6): 1016-1021.

11 Zumkeller W, Orth U, Gal A. Three novel PAX6 mutations in patients with aniridia. Mol Pathol 2003; 56(3): 180-183.

12 Nishina S, Kohsaka S, Yamaguchi Y, Handa H, Kawakami A, Fujisawa $\mathrm{H}$ et al. PAX6 expression in the developing human eye. Br J Ophthalmol 1999; 83(6): 723-727.

13 Davis JA, Reed RR. Role of Olf- 1 and Pax-6 transcription factors in neurodevelopment. J Neurosci 1996; 16(16): 5082-5094.

14 Treisman JE. How to make an eye. Development 2004; 131(16): 3823-3827.

15 Glaser T, Jepeal L, Edwards JG, Young SR, Favor J, Maas RL. PAX6 gene dosage effect in a family with congenital cataracts, aniridia, anophthalmia and central nervous system defects. Nat Genet 1994; 7(4): 463-471.
16 Baulmann DC, Ohlmann A, Flugel-Koch C, Goswami S, Cvekl A, Tamm ER. Pax6 heterozygous eyes show defects in chamber angle differentiation that are associated with a wide spectrum of other anterior eye segment abnormalities. Mech Dev 2002; 118(1-2): 3-17.

17 Ramaesh T, Collinson JM, Ramaesh K, Kaufman MH, West JD, Dhillon B. Corneal abnormalities in Pax6 + / - small eye mice mimic human aniridia-related keratopathy. Invest Ophthalmol Vis Sci 2003; 44(5): 1871-1878.

18 Azuma N, Nishina S, Yanagisawa H, Okuyama T, Yamada M. PAX6 missense mutation in isolated foveal hypoplasia. Nat Genet 1996; 13(2): 141-142.

19 Dominguez M, Ferres-Marco D, Gutierrez-Avino FJ, Speicher SA, Beneyto M. Growth and specification of the eye are controlled independently by Eyegone and Eyeless in Drosophila melanogaster. Nat Genet 2004; 36(1): 31-39.

20 Azuma N, Tadokoro K, Asaka A, Yamada M, Yamaguchi Y, Handa $\mathrm{H}$ et al. The Pax6 isoform bearing an alternative spliced exon promotes the development of the neural retinal structure. Hum Mol Genet 2005; 14(6): 735-745. 